\title{
FEASIBILITY STUDY AND MODELING OF COMPONENTS FOR AN INFORMATIONAL SPACE SYSTEM BASED ON A LARGE DIFFRACTIVE MEMBRANE
}

\author{
V.V. Salmin ${ }^{1}$, S.V. Karpeev ${ }^{2}$, K.V. Peresypkin ${ }^{1}$, A.S. Chetverikov ${ }^{1}$, I.S. Tkachenko ${ }^{1}$ \\ ${ }^{1}$ Samara National Research University, Samara, Russia \\ ${ }^{2}$ Image Processing Systems Institute - Branch of the Federal Scientific Research Centre "Crys- \\ tallography and Photonics" of Russian Academy of Sciences, Samara, Russia
}

\begin{abstract}
The paper presents a feasibility study for an optical system, based on a diffractive membrane. The authors have analyzed structural design of diffractive optical elements for space application and developed finite element models of diffractive lens's carrier and mount. The models were developed for two different schemes: MOIRE project and for the original scheme, developed by the authors. The schemes were analyzed for structural stiffness of the diffractive lens's carrier and mount. The shapes and frequencies of natural oscillations of the carriers of the lens were calculated. The problems of the membrane system's orbit injection and operation were analyzed. An algorithm for the terminal control of the orbital period, eccentricity and longitude of the point of standing of the orbital surveillance system was proposed.
\end{abstract}

Keywords: diffractive optics, space membrane optical system, finite element modeling, space telescope, geostationary orbit

Citation: Salmin VV, Karpeev SV, Peresypkin KV, Chetverikov AS, Tkachenko IS. Feasibility study and modeling of components for an informational space system based on a large diffractive membrane. CEUR Workshop Proceedings, 2016; 1638: 132-148. DOI: 10.18287/1613-0073-2016-1638-132148

\section{Introduction}

The problem of high-quality Earth's surface sensing using spacecraft (SC) is of great importance. Modern SC, used for remote Earth sensing (RES), utilize big lenses or mirrors as light-harvesting elements. It leads to a drastic increase in mass and size characteristics of those SC.

Modern RES SC operate on low orbits, hindering their performance. For example, they cannot be used to perform continuous shooting. Also, their position might not allow them to shoot a particular area. In order to perform continuous shooting the satellite must be delivered to the geostationary orbit (GSO). 
Since GSO RES SC require high orbits (about $36000 \mathrm{~km}$ ), obtaining high-resolution images requires special optics. Refractive or reflective optics, made of quartz or sitall, is relatively heavy; therefore, it is problematic to launch new RES SC.

The US Agency for Defense Advanced Research Projects DARPA is working of the project of a new space telescope with a membrane diffractive optical system. The project is called MOIRE or Membrane Optical Imager for Real-Time Exploitation [13].

The MOIRE project plans to use a polymer membrane to create optical elements. The diameter of the entrance pupil will be about 10-20 m (Fig. 1a). Unlike regular lenses or mirrors, membrane is a diffractive optical element (DOE). Phase bandwidth of a DOE is formed by microscopic concentric grooves engraved on a plastic surface. The width of these grooves varies from a few hundred to four micrometers. Optical membranes are much less effective than the traditional optical elements, but, since there are no more size constraints, the entrance pupil and relative aperture ratio can be increased significantly, while reducing or maintaining SC mass. Membranes will be mounted on thin metal blades (Fig, 1b), that would fold, forming a SC 6 meters wide.
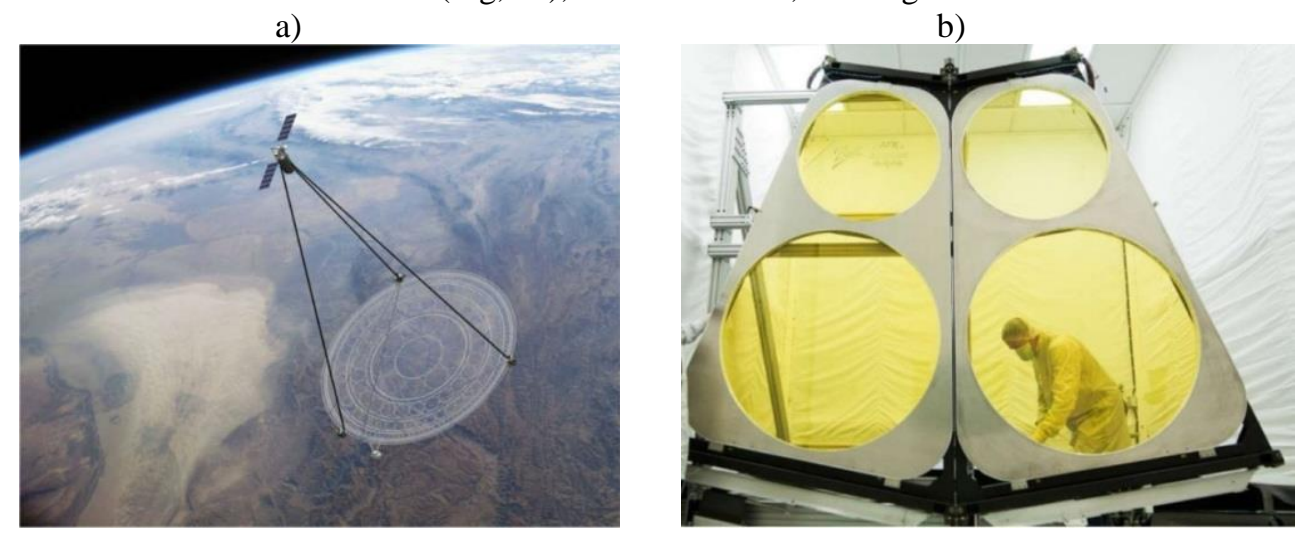

Fig. 1. Membrane Optical Imager for Real-Time Exploitation project [1-3]

Diffractive optics has a number of advantages over refractive optics on such key parameters as the weight and size. Aso, it enables simple elements replication in order to form both spherical and aspherical wavefront sets, making diffractive optic's elements useful for different applications. The majority of studies consider diffractive optical elements either as additional elements for the compensation of aberrations of a refractive imaging system [4, 5], or as elements for focusing on a particular area [4-14]. For example, in $[6,7]$ longitudinal intensity distribution for a coherent case is considered, [8] considers the process of a plane wave focus using a Fresnel lens, and [9] researches methods of compensation of DOE chromatic aberration.

There are considerably less studies on application of diffractive optics towards image forming. The well-known problem of DOE chromatic aberration hinders its spectrl range and lowers the image quality in polychromatic light.

The article presents the results of a feasibility study for a space diffractive membrane optical system (SDMOS), and a study of structural stiffness of SDMOS that performs remote Earth sensing using membrane diffractive optical elements (DOE). 


\section{Possibilities of chromatic aberration's correction in space telescopes using DOE}

Obvious requirements for space telescopes are the correction of chromatic aberrations and sufficient spatial resolution at a relatively small (a few degrees) angular field.

At first glance, it seems that the diffractive optical elements, due to their extremely large chromaticity can be of little help in solving the above-mentioned tasks. And indeed, technoogical constraints on manufacturing high-aperture DOE do not alow creation of high-aperture diffraction achromatic object lens with required characteristics (which would be an ideal case in terms of size and weight characteristics). Hybrid diffractive-refractive optical systems attracted attention of researches [5, 15], due to their ability to effectively correct chromatic aberrations. However, from the point of utilizing the greatest advantage of diffractive optics - its relative low weight, those systems are a compromise, since they include big and therefore heavy, refractive elements.

In case of placing the telescope on a geostationary satellite, the aperture might be sacrificed due to the possible increase in exposition. However, even in case of low aperture, creation of purely diffractive optical systems faces problems, since it is impossible to correct chromatic aberration by combining collecting diffractive lenses, distributing the optical power onto several elements. Known approach to correcting chromatism by combining two purely diffractive lenses with different optical powers [16] increases the optical powers of the lenses compared with an equivalent single lens that causes additional technological problems. It is worth noting that a classic way to deal with chromaticism - a transition to the reflective optics for diffractive elements is not applicable for obvious reasons. However, conventional optical elements in the objective lens could be made reflective, therefore removing the chromaticity problem.

Nowadays, the most promising approach is to develop a diffraction mirror imaging system in which suppression of chromaticity would be done by the imaging system architecture, as well as modification of the structure of the DOE itself. An example of such a system is presented in [17] (Figure 2), that incudes a correcting DOE 3 on top of the "photonic grid" 1 . The correcting DOE 4 is $40 \mathrm{~mm}$ in diameter with the focal distance of 158,5 mm. Focal distance of the lens 2 is $400 \mathrm{~mm}$. Mirrors 4 and 5 are 200 $\mathrm{mm}$ in diameter and focal distances 734 and $807.7 \mathrm{~mm}$ respectively. The frequency bandwidth of such a system is about $40 \mathrm{~nm}$.

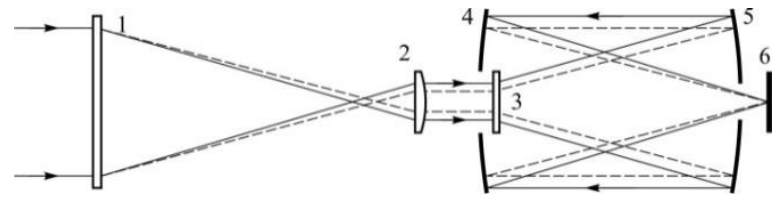

Fig. 2. The optical scheme of a mirror - diffraction telescope, based on diffractive doublet: 1 "photonic grid", 2 - matching lens, 3 - correcting DOE, 4, 5, - mirrors, 6 - image plane 


\section{Determining the structural stiffness of the diffractive lens carrier frame for a MOIRE spacecraft}

For the MOIRE project, the structure, that is attaching the lens to the spacecraft (or the lens carrier), consists of three straight foldable frames. One end of the frames is connected to the body of the satellite, the other - to the Fresnel lens carrier (Figure 3). We took this design as a prototype. We model the rods of the frame as a unidirectional carbon fiber.

According to the analysis of the diffractive lens' weight in comparison to the traditional primary telescope lenses presented in [1-3], in average diffractive lens is about seven times ligher then a mirror of the same area.

The diffractive lens for the SDMOS is estimated to weight $158.5 \mathrm{~kg}$. The design of the SDMOS is characterized by the fact that its optical system should receive a focused and not a plane-parallel lightstream.

The MOIRE project lens in the unfolded state is a flat frame. This frame is formed by three concentric rings, connected by radial beams (Figure 3). The perforated membranes, forming the diffractive lens, are stretched on frames, formed by adjacent radial beams and sections of adjacent rings. Radial beams separate the lens onto sections that are attached to each other pivotally. In the folded state the sections form a bellows shape. During the process of unfolding, sections rotate relatively to each other until the lens becomes flat. After the unfolding, the pivets get locked.

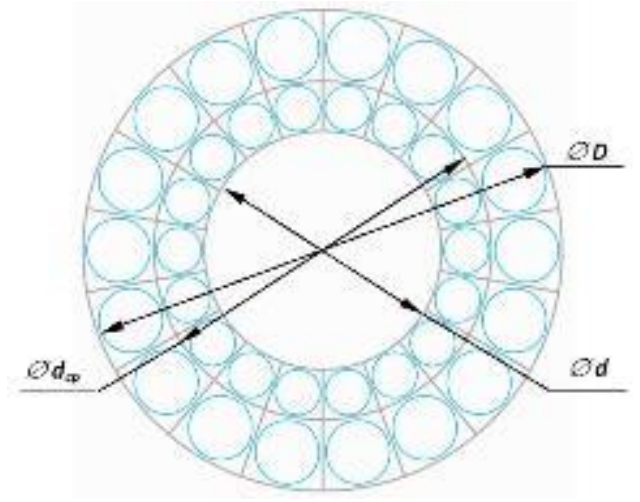

Fig. 3. Lens structure $\left(D=10 \mathrm{~m} ; d=4.96 \mathrm{~m} ; d_{c p}=7.04 \mathrm{~m}\right)$

In order to ensure that the lens is not going to deform from reaction with the carrier, the lens must be attached to it in a statically determinable way. To achieve this, the carrier frames must be held together by a special structure (the mount) and not the lens itself. The lens is supposed to be fastened to this mount.

Structure, presented on the Figure 4, corresponds to the published sheme of the MOIRE project, exept for the special lens mount. This mount is a triangular frame, the supporting frames are fixed to the tops of the triangle. The lens is fixed to the middle parts of the frame's beams. The structure is fixed in the longitudinal and circumferential extent of freedom and is statically determinable. 
Stiffness of the lens moubt is determined by the stiffness of extended elements of the spacecraft's structure. For the considered design such elements are the lens carrier, lens frame and the foldable frames. A finite-element model was created to determine the stiffness of the lens mount. The structural elements are modeled with beam finite elements.

Sturctural material is unidirectional carbon fiber.

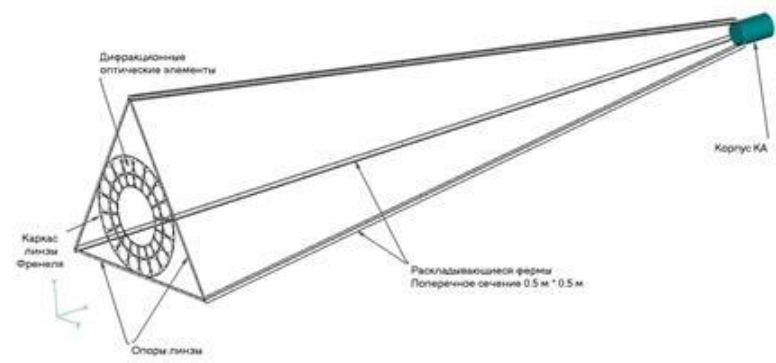

Fig. 4. Lens mount structure for the MOIRE spacecraft. The embodiment with straight bearing frames

To determine structural self-oscillation using finite-element method, the following problem must be solved $[18,19]$ :

$\left(-\omega_{i}^{2} \cdot[M]+[K]\right) \cdot\left\{U_{i}\right\}=0$,

where $\omega_{i}$ - $i$-th rotational frequency; $[M]$ - mass matrix; $[K]$ - stiffness matrix; $\left\{U_{i}\right\}-i$-th eigenform. The solution is carried out for a few lower tones of natural oscillations by the method of Lanczos. Stiffness matrix of the elastic system within the finite element method has the following form:

$$
[K]=\sum_{k=1}^{N e} \int_{V e k}[B]_{k}^{T} \cdot[D]_{k} \cdot[B]_{k} d v,
$$

where $\mathrm{Ne}$ - the number of finite elements; Vek - volume of the $k$-th finite element; $[D]_{k}$ - Hooke matrix for the $k$-th finite element; $[B]_{k}$ - connection matrix between angular displacements and deformations: $\{\varepsilon\}_{k}=[B]_{k} \cdot\{u\}_{k} ;\{u\}_{k}$ - nodal displacements of the $k$-th finite element; $\{\varepsilon\}_{k}$ - deformation of the $k$-th finite element. Koefficients of the $[B]_{k}$ matrix can be found by differentiating functions of form $[\Phi]_{k}$ for finite elements for corresponding coordinates. The mass matrix of the elastic system within the finite element method has the following form:

$$
[M]=\sum_{k=1}^{N e} \rho_{k} \cdot \int_{V e k}[\Phi]_{k}^{T} \cdot[\Phi]_{k} d v,
$$

where $[\Phi]_{k}$ - form function of the k-th finite element: $\{u(x)\}_{k}=[\Phi]_{k} \cdot\{u\}_{k} ;\{x\}$ coordinates of a point within a finite element; $\rho_{k}$ - material density for the $k$-th finite element. 
We found the first ten elastic forms of natural oscillations. The frequency of natural oscillations of the lower tone, the movement by which distorts the image, is 0.0144 $\mathrm{Hz}$ (Fig. 5). This value of the natural frequency is too low to hope that it will be possible to quickly direct CDMOS of the MOIRE scheme onto the object. One oscillation period on this tone is 69 seconds. If the structure's oscillations, caused by inertial forces during direction process, will be damped after 100 periods, it will take almost two hours to stabilize the system before taking a picture. Practically, it means that this satellite can only be used to observe static objects from a geostationary orbit. Even in this case distortions will occur after the slightest alterations of the orbit. In order to solve this problem, the stiffness of the structure must be drastically increased.

Low stiffness of the MOIRE project frame is due to the large length of carrying frames relative to their transverse dimensions. These kinds of frames naturally have low transverse rigidity.

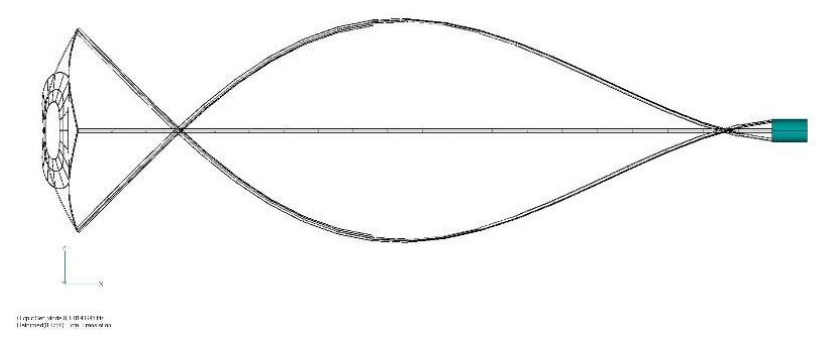

Fig. 5. Second elastic form of natural vibrations of the lens frame for a MOIRE sheme satellite (natural frequency $0,0144 \mathrm{~Hz}$ )

\section{Study of stiffness and load bearing capacity of a satellite's diffractive lens frame, laced by cables}

\subsection{Study of frame structural stiffness}

We examine an alternative structure layout, where the load bearing frames are arkshaped. The bending radius of load-bearing frames is set to $60 \mathrm{~m}$. Longitudinal cables connect the ends of frames and prevent the arks from changing radius. Due to the ark shape of the frames, lateral forces in the ark planes will cause compressive strain in the frames rather than bending (that would accure for the straight frames). This dramatically increases the lateral stiffness of the frames in the arch planes. Increased transverse stiffness makes it possible to connect pairs of frames with cables. With sufficient cable's tightening tension they would work like rods. These cables connect thre frames into one large frame with high transverse stiffness (Fig. 6).

The proposed lens frame structure is a three -beam star.

Finite element model for this structure has additional rod elements that represent the strained cables. The finite-element mesh configuratiuon of the lens frame corresponds to the altered structure of this element. 


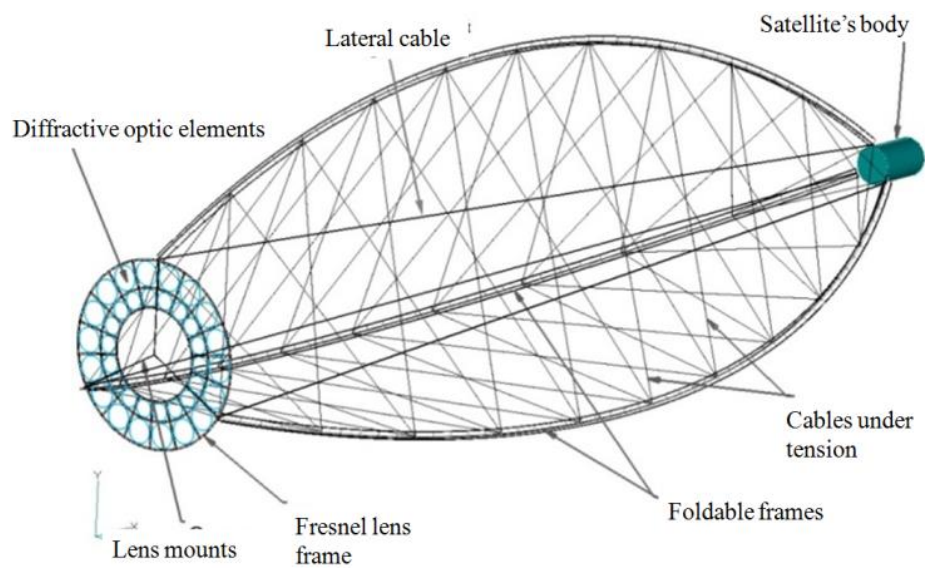

Fig. 6. Diffractive lens frame structure. The structure variant with cable fastening

We found the lowest tones of natural oscillations. The frequency of the lowest tone of natural oscillations, the movement by which distorts the image, is $1,48 \mathrm{~Hz}$ (Fig. 7). This natural frequency is similar to the natural frequency of solar panels of traditional surveillance satellites. It means that the CDMOS with this lens frame layout coud be operated in the same manner as traditional surveillance satellites, provided the gyro system has enough power. Therefore, the use of arch frames, fastened by cables, can satisfactory solve the frame stiffness problem.

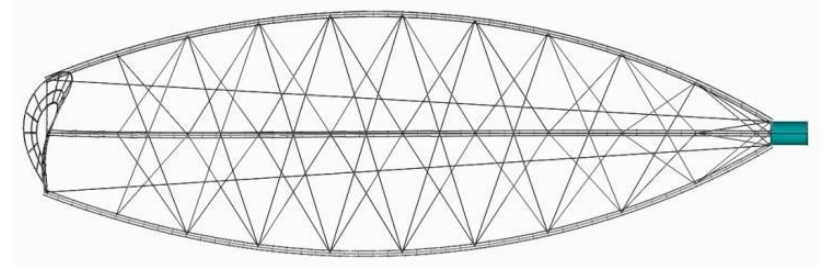

a)

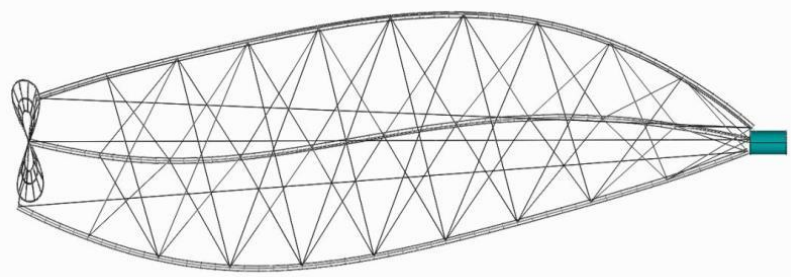

b)

Fig. 7. Lover tones of natural frequencies of the Fresnel lens, fastened by cables: a - fifth elastic form of natural vibrations (frequency of $1.48 \mathrm{~Hz}$ ), b - eighth elastic form of natural vibrations (frequency of $2.06 \mathrm{~Hz}$ ) 


\subsection{Study of the load-bearing capacity of the lens frame structure}

In order for the proposed structural layout to work, the cables must remain strained during the satellite's maneuvers. This means that the cable pre-tension must be higher than the tension easing from the structure's deformation during maneuvers. The finite-element model takes this tension easing into account by addition of compressing forces inside of the rods that represent the cables. In order to determine the cable pre-tension values, the satellite's turn must be modeled. Maximum calculated straining force in the rods would be the desired value of the pre-tension force.

The satellite is turning at an angle $\varphi_{P}=5^{\circ}$ in a time $t_{P}=60 \mathrm{~s}$ around the direction perpendicular to the optical axis of the satellite. The law of the control torque change is taken in form of two successive rectangular pulse pairs of forces in different directions (Fig. 8), which corresponds to the swiftest turn.

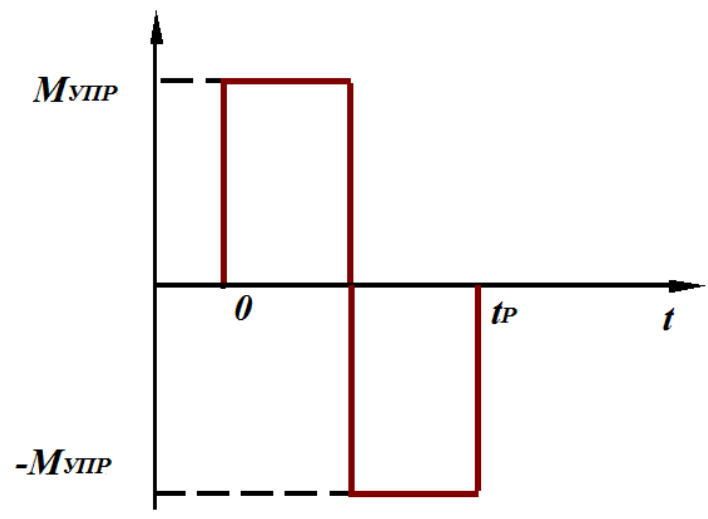

Fig. 8. The law of the control torque change in form of two successive rectangular pulse pairs of forces in different directions

During the satellite's turn it rotates around an inertial coordinate system. The structure of the satellite is elastically vibrating. The amplitudes of those vibrations are expected to be low in the sence that they will not substantially affect inertial and elastic properties of the satelilite's structure. If we consider the satellite in the associated coordinate system, there will be no geometric nonlinearity. Modeling is performed in the inertial coordinate system in order to simplify the tasks of applying boundary conditions and analyzing the results. In this case, in order to turn the structure's finite-element model, recalculations of matrix of mass, dampling and structural stiffness for the new spatial position are needed. However, the turn angle is small and therefore the matrixes do not change significantly. It means that we can neglect the influence of rotation of the model onto the matrix coefficients and analyze the system as linear.

Movement over time is found using linear transition analysis in MSC Nastran. This analysis is performed by integrating the main dynamic equation over the time [20]. $[M] \cdot\{\ddot{u}\}+[C] \cdot\{\dot{u}\}+[K] \cdot\{u\}=\{P(t)\}$, where $\{P(t)\}$ - nodal force vector; $j$ - increment number; $\{u\}$ - nodal displacement vector $\{\dot{u}\}$ - nodal velocity vector; $\{\ddot{u}\}$ - nodal acceleration vector; $[C]$ - damping 
matrix. Speed and acceleration are expressed through movement by the centraldifference approach:

$$
\{\ddot{u}\}_{j}=\frac{\{\dot{u}\}_{j+1}-\{\dot{u}\}_{j-1}}{2 \cdot \Delta t} ;\{\dot{u}\}_{j}=\frac{\{u\}_{j+1}-\{u\}_{j-1}}{2 \cdot \Delta t},
$$

where $\Delta t$ - time increment. Then, by averaging the vector of nodal forces on three adjacent steps of integration in time, the system (1) is transformed into:

$$
\left[A_{1}\right] \cdot\{u\}_{j+1}=\left[A_{2}\right] \cdot\{u\}_{j}+\left[A_{3}\right] \cdot\{u\}_{j-1}+\left[A_{4}(t)\right] \text {. }
$$

Calculation of the displacement using the system of linear equations (2) carried out for the initial conditions $\{u\}_{j}=0$ и $\{u\}_{j-1}=0$, which corresponds to a fixed CDMOS in the initial time frame. Found displacement-time dependancies are presented on Figure 9. The first 60 seconds of test time correspond to reversal of CDMOS. In the next 40 seconds there is a gradual damping of the oscillations arising during the turn.

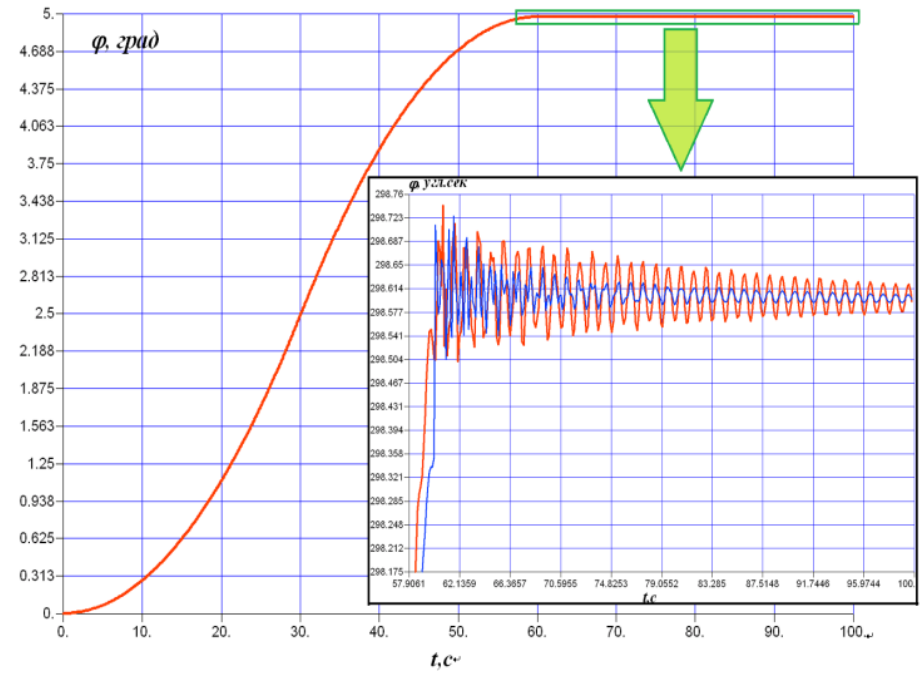

Fig. 9. The rotation angles of the mirror (red line) and the spacecraft body (blue line) based on the results of the finite element modeling of rotation

The resulting dependences of maximum stresses in the cables due to reversal is shown in Figure 10. As can be seen from this figure, cable tightness of $0.25 \mathrm{MPa}$ provides the necessary tension during the whole reversal process.

The tension of the longitudinal cables is determined by the task of deployment of the structure after injection into orbit and is set to $100 \mathrm{MPa}$. Tension of the longitudinal cables causes the the arch frames to bend and tighten the cables between the frames. It is necessary to check whether this tension will be sufficient to maintain the cables tension during spacecraft's revolutions.

In order to check it, the finite element analysis of deformation of the lens mounting structure under the action of the tension of the longitudinal cables was performed. The linear static analysis was performed in MCS Nastran. This analysis is a solution of 
the linear system of the Hooke's law equations for the considered, with respect to the vector of nodal displacements $\{u\}$ :

$[K] \cdot\{u\}=\{P\}$.

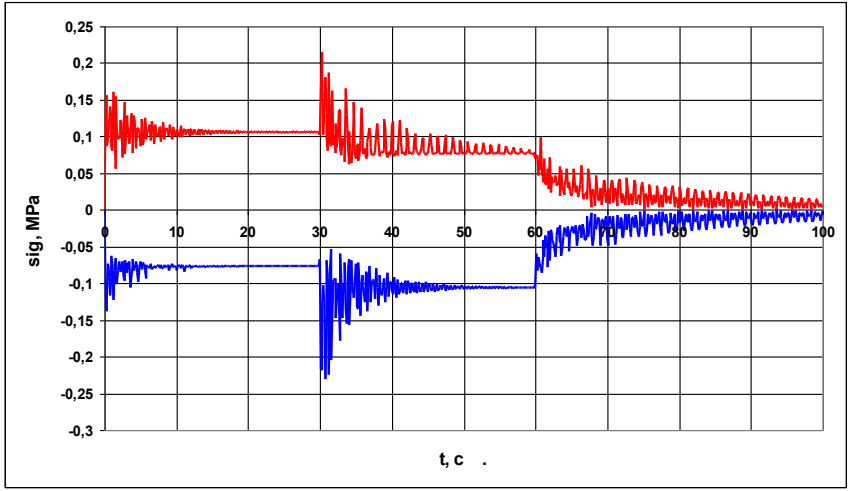

Fig. 10. Maximal changes in stresss of cables, connecting the frames, depending on the time

Deformation of structure from the effects of tighteing of longitudinal cables is shown in Figure 11. Figure 12 shows tightness of cables between the arch frames. According to figure 12 , the cable tightness does not exeed $0.85 \mathrm{MPa}$, which is below the minimal cable tension during satellite's maneuvres.

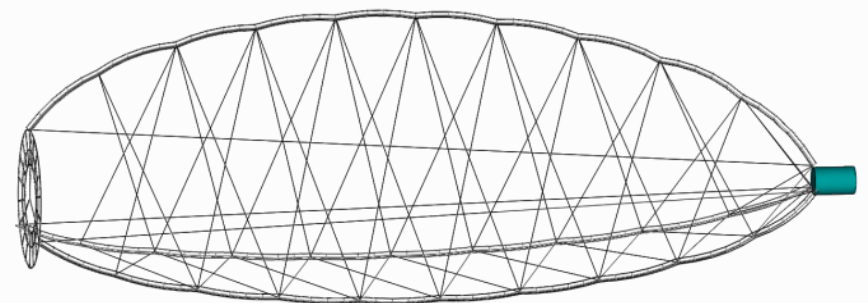

Fig. 11. Structural deformation under the cables tension. The distance between the lens and body of the spacecraft has decreased by $6.9 \mathrm{~mm}$

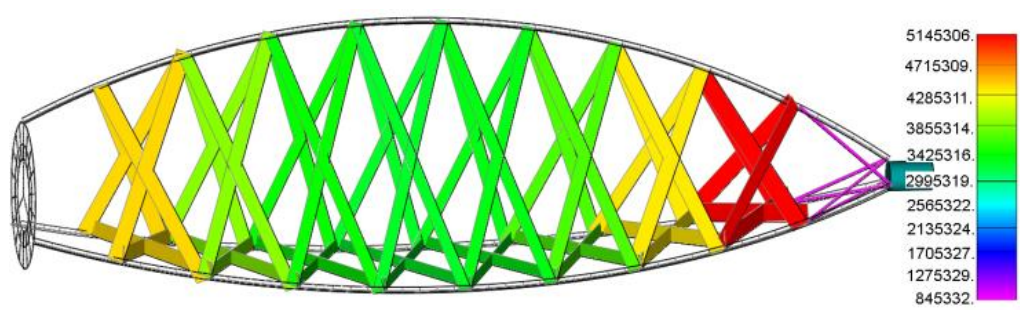

Fig. 12. Cables tension between the arch frames. Maximum stress $5,1 \mathrm{MPa}$, minimum stress $0.85 \mathrm{MPa}$ 


\section{CDMOS launch to a geostationary orbit and the correction of the satellite's orbit}

\subsection{Ballistioc sheme of the CDMOS geostationary orbit delivery}

CDMOS based on a big diffractive membrane is a large object with low structural stiffness. During orbit injection of such a system using a chemical booster, significant overloads may arise, possibly leading to undesirable structural changes of the diffractive optical system.

In this case, low-thrust electric propulsion engines seem to be better suited for the task of the CDMOS's geostationary orbit delivery. Electric propulsion engines create accelerations of about $0,5 . .1,0 \mathrm{~mm} / \mathrm{s}^{2}$. Transportation from a low Earth orbit to the geostationary orbit will take from 100 to 200 days. Weight of a space system, including tugboat with solar electric propulsion CDMOS will be from 6000 to $8000 \mathrm{~kg}$. Weight of the surveillance satellite, based on a diffractive membrane, is estimated to be about $3500 \mathrm{~kg}$, the lens itself (including diffractive membranes, frame and mount of the lens) would weight $500-600 \mathrm{~kg}$. Thus the required thrust of the propulsion system would be from 2.5 to $4 \mathrm{~N}$ and the required power for the electric propulsion engine would be $50-70 \mathrm{~kW}$.

Ballistic scheme of CDMOS launch to a geostationary orbit includes several steps. On the first step the orbital system, consisting of folded CDMOS and the space tugboat is delivered to the low Earth's orbit by a middle-class rocket "Soyuz" or "Angara". On the next stage the payload assist module delivers the system to a higher orbit (500-600 $\mathrm{km}$ ), where the atmospheric influence is negligeable. On this temporary orbit space tugboat's solar panels and the membrane system are unfolded. On the final step of the orbit injection CDMOS is delivered into the geostationary orbit to the stand point by the solar space tugboat with an electric propulsion engine.

\subsection{Dynamics of the CDMOS's geostationary orbit delivery}

Low-thrust flights between non-complanar circular orbits are controlled by the angle between the direction of the transversal and thrust vector $\psi$ (Fig. 13).

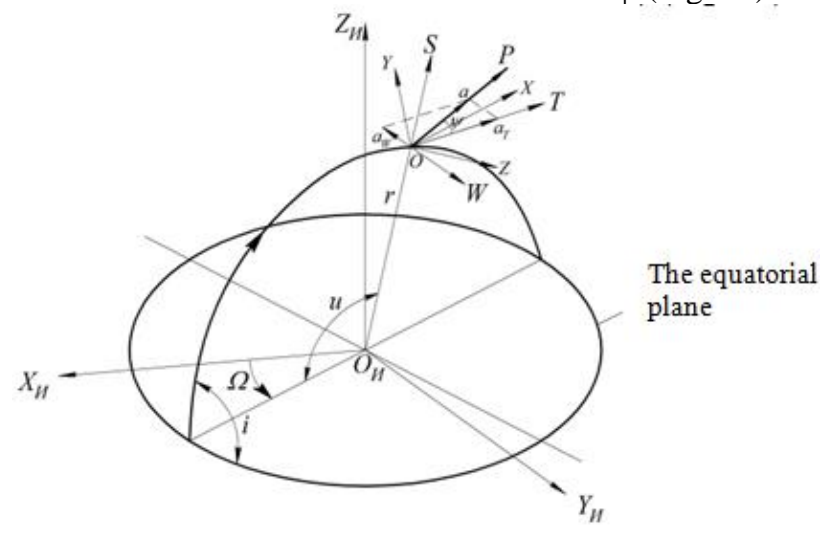

Fig. 13. The position of the orbit and thrust vector control scheme 
Then, the projections of the thrust acceleration on the orbital axis of the coordinate system are:

$a_{T}=\frac{P}{M} \cos \psi, a_{S}=0, a_{W}=\frac{P}{M} \sin \psi$,

where $P$ - electric propulsion engine's thrust, $M$-current mass.

Additionaly, thrust vector control for flights between non-complanar orbits requires a change in the sign of the binormal component of reactive acceleration $a_{W}$ twice per revolution [21].

In [22] a simplified satellite's low-thrust movement model optimal control of the $\psi$ is defined as:

$\psi\left(V_{X}, u\right)=\psi_{m}\left(V_{X}\right) \operatorname{sign}(\cos u)$,

where $\psi_{m}\left(V_{X}\right)=\operatorname{arctg}\left\{A \cdot\left[1-B-\frac{V_{X}}{V_{0}} \cdot C\right]^{-1}\right\}-$ the amplitude of the periodic oscil-

lations of the $\psi$ angle;

$$
\begin{gathered}
A=\sqrt{\frac{r_{0}}{r_{K}}} \sin \pi \frac{i_{K}-i_{0}}{2}, B=\sqrt{\frac{r_{0}}{r_{K}}} \cos \pi \frac{i_{K}-i_{0}}{2}, \\
V_{0}=\sqrt{\frac{\mu}{r_{0}}}, C=\sqrt{1-2 \sqrt{\frac{r_{0}}{r_{K}}} \cdot \cos \pi \frac{i_{K}-i_{0}}{2}+\frac{r_{0}}{r_{K}}},
\end{gathered}
$$

$\mu$ - Earth's gravitational parameter, $V_{X}=\int_{0}^{t} a d t$ - characteristic velocity, $u$ - argument of latitude (the phase angle in the orbital motion), $r, i$ - the current values of the average radius vector of the satellite and the inclination of the orbit.

Calculations, disclosed in [19], show analytical solutions that describe flight trajectory between non-complanar orbits within the averaged motion model. This model does not depend on the spacecraft's design charachteristics (Figure 14). As shown on Figure 14, the orbit radius $r$ first increases from the initial value $r_{0}$ to the maximum and then decreases (braking section) to a value $r_{K}$. The $\psi$ angle is more than $\frac{\pi}{2}$. The value $\psi=\frac{\pi}{2}$ is achieved in the highest point $\left(r=r_{\max }>r_{\Gamma C O}\right)$. This peculiarity of the trajectory is caused by the fact that it is more energetically effective to change the inclination of the orbit on the higher orbits.

\subsection{Maintaining the CDMOS's functioning orbit and the standing point's longitude}

During operation of the CDMOS based on a large diffractive membrane it would be affected by lunar-solar disturbances and considerable solar pressure. Those effects will cause the satellite to change its orbital parameters from the nominal. The first altered characteristics would be the period and geographical longitude of the standing point of the geostationary orbit. Therefore the orbital period and the longitude of the point of standing should be constantly corrected. 


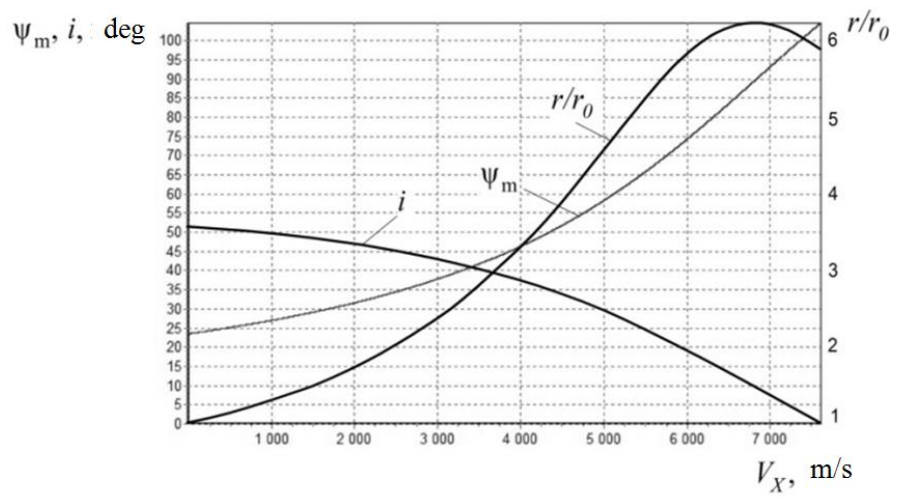

Fig. 14. The change of trajectory parameters and control parameters during flight $\left(\mathrm{r}_{0}=7171 \mathrm{~km}, \mathrm{r}_{\mathrm{K}}=42164 \mathrm{~km}, \mathrm{i}_{0}=51,6^{0}, \mathrm{i}_{\mathrm{K}}=0^{0}\right)$

The work [23] describes a discrete model of the motion of a geostationary spacecraft under the influence of small transversal acceleration. Solving of the optimization problem with the classical dynamic programming method [24] is difficult, because the discrete movement model is non-linear. Therefore, in [23] the authors propose an approximate method for solving the problem based on the three-step control algorithm for circulation period, eccentricity and longitude of the point of standing. Orbit correction is carried out using low-thrust electric rocket engine that produces acceleration in the transversal direction.

The essence of the algorithm is as follows. We assume that the control structure consists of three active regions $\mathrm{AB}, \mathrm{CD}$, EO (Fig. 15) with respective durations $\tau_{0}, \tau_{1}, \tau_{2}$ and two passive regions with respective durations $t_{\Pi 1}$ и $t_{\Pi 2}$. On the $\mathrm{AB}$ region eccentricity decreases to zero, On the $\mathrm{CD}$ region eccentricity increases until it reaches the value $e$ ', then on the EO region eccentricity decreases to zero once again.

This control structure guarantees the delivery of the spacecraft, equipped with a lowthrust engine, to the target poin of a geostationary orbit.

Discrete model of plane motion of geostationary satellites under the influence of small transversal acceleration is presented in as [23]:

$$
\begin{aligned}
& \Delta T(k+1)=\Delta T(k)+3 a_{\mathrm{T}}\left(T_{3}+\Delta T(k)\right) \sqrt[3]{\frac{T_{3}+\Delta T(k)}{2 \pi \cdot \mu}} \tau(k), \\
& \Delta \lambda(k+1)=\Delta \lambda(k)+\left(\frac{2 \pi}{T_{3}+\Delta T(\mathrm{k})}-\omega_{3}\right) \cdot\left(t_{\Pi}(k)+\tau(k)\right), \\
& \Delta e(k+1)=\left|\Delta e(k)-2 \cdot a_{T}\left(\frac{T_{3}+\Delta T(k)}{2 \pi \cdot \mu}\right)^{1 / 3} \tau(k)\right|,
\end{aligned}
$$

where $k=0, \ldots, N-1, t_{\Pi}(k)$ is determined by formulas $t_{\text {п }}=\frac{T_{0}}{2}(1+2 m)-\frac{\tau}{2}-\frac{\vartheta_{0} T_{0}}{2 \pi}$ for $a_{T}>0$, 


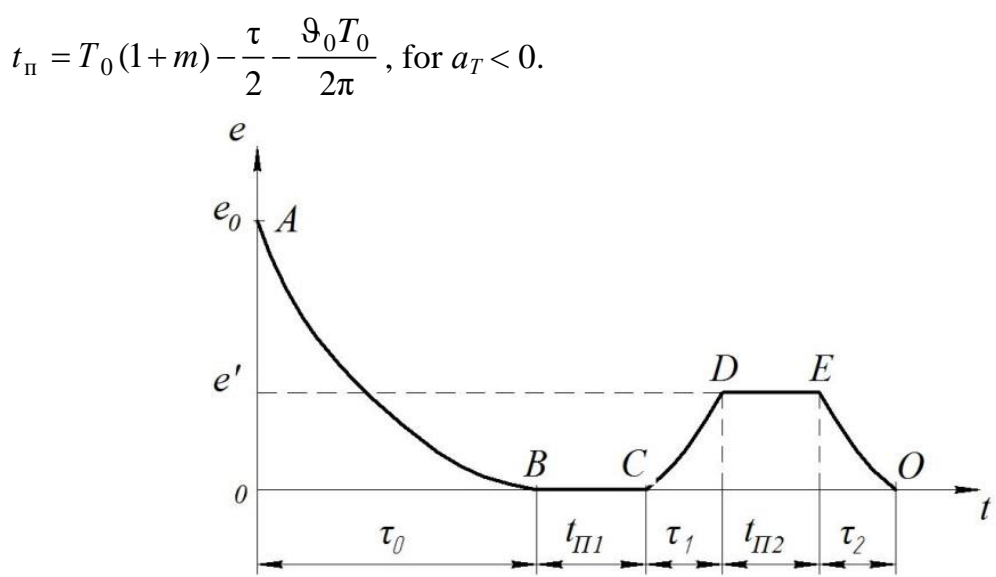

Fig. 15. The change of eccentricity with three-step control structure

Functional

$I=\Delta X_{K}^{T} \Lambda \Delta X_{K} \rightarrow \min$.

Where $a_{T}$ - transversal acceleration, $\vartheta_{0}$ - true anomaly angle before correction; $\Delta X_{K}$ $=\left\{\Delta T_{K}, \Delta \lambda_{K}, \Delta e_{K}\right\}^{T}$ - the final state vector, where $\Delta T_{K}=T_{K}-T_{3}, \Delta e_{K}=e_{K}-e_{\Gamma C O}$, $\Delta \lambda_{K}=\lambda_{K}-\lambda_{P} ; T_{K}, e_{K}, \lambda_{K}$ - values of the orbital period, eccentricity and longitude of the satelite's standing point on the orbit at the end of the correction maneuver; $T_{3}-$ circulation period of the spacecraft in geostationary orbit, equal to a star day $T_{3}=$ $86164,09 \mathrm{c} ; e_{\Gamma C O}$-eccentricity of the geostationary orbit; $\lambda_{P}-$ longitude of the working point of standing of a satellite; $\Delta T_{0}=T_{0}-T_{3}, \Delta e_{0}=e_{0}-e_{\Gamma C O}, \Delta \lambda_{0}=\lambda_{0}-\lambda_{P}$, where $T_{0}, e_{0}, \lambda_{0}$ - values of the orbital period, eccentricity and longitude of the point of standing on the orbit before the spacecraft correction maneuver.

Based on the proposed control structure an analytical solution is obtained for the $\tau_{0}$, $\tau_{1}, \tau_{2}, t_{\Pi 1}, t_{\Pi 2}[23]$.

Examples of satellite's phase trajectories are given for the found solution for two cases: initial positive deviation of period $(\Delta \mathrm{T} 0>0)$ (Fig. 16a) and the initial negative deviation of period $(\Delta \mathrm{T} 0<0)$ (Fig. 16b).

The presented algorithm has shown high accuracy in modeling of the correction of orbit for a surveillance satellite, fitted with an electric propultion engine. For example, for $\Delta \mathrm{T}_{0}=1000 \mathrm{~s}, \mathrm{e}_{0}=0,005, \Delta \lambda_{0}=0,087 \mathrm{rad}$ the final orbit parameters deviations were: orbital period $\Delta T_{K}=1,3 \mathrm{~s}$, standing point longitude $\Delta \lambda_{K}=0,15^{\circ}$, eccentricity $\Delta e_{K}=1 \times 10^{-4}$. Durations of the active and passive sections were $\tau_{0}=7758 \mathrm{~s}, \tau_{1}=1997$ $\mathrm{s}, \tau_{2}=1998 \mathrm{~s}, t_{\Pi 1}=260200 \mathrm{~s} \approx 3$ days, $t_{\Pi 2}=40170 \mathrm{~s} \approx 0,46$ days. 
a)
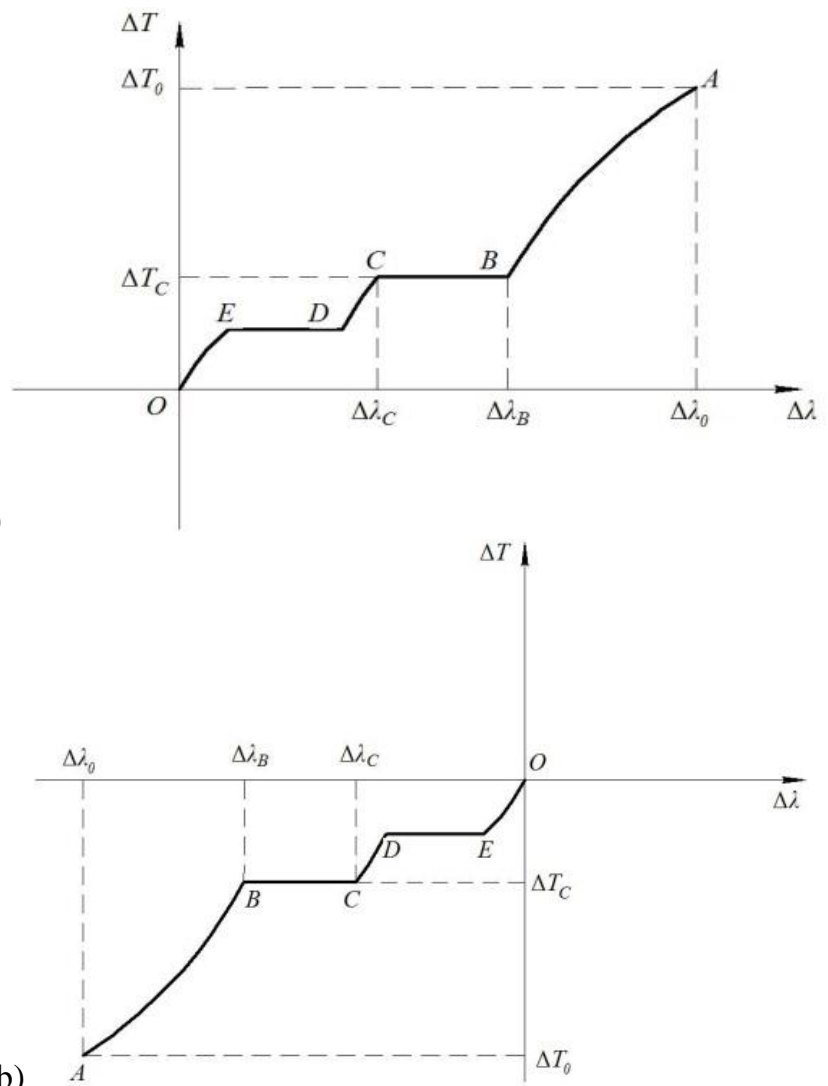

Fig. 16. Examples of satellite's phase trajectories: a - for $\Delta \mathrm{T}_{0}>0, \mathrm{~b}$ - for $\Delta \mathrm{T}_{0}<0$

\section{Conclusion}

The feasibility study for a diffractive imaging system has been carried out. Creation of a purely diffractive optic system currently is practically impossible because of the difficulty of correcting chromatic aberration of the image. For space-based telescopes, located on the geostationary orbit, a promising approach is the use of diffractionrefraction imaging systems, in which the chromaticity suppression would be done by the imaging system's architecture, as well as modifications of the structure of the diffractive optic elements.

A structural finite-element analysis of the space telescope, utilizing diffractive membranes as main elements of the optical system, has been carried out. The analysis was performed for two schemes - one for the MOIRE prototype and the other for the original scheme, proposed by the authors. In the original scheme the frames that hold the diffractive membrane ( 10 meters in diameter), are made in the ark forms and laced with cables. As a result of the analysis, the forms and frequencies of natural oscillations of the mount of the lens (the frame) were obtained. The frequency of natural 
oscillations of the lower tone, movement by which distorts the image, is greater by two orders of magnitude for the proposed scheme in comparison with the prototype. The use of arch-shaped frames and cable lacing in structures of CDMOS, using diffractive membranes as main components of the optical system, allows achieving sufficient structural stiffness.

A low-thrust electric propulsion engine is proposed as a preferable means of CDMOS delivery from the lower orbit to the point of destination on the geostationary orbit.

\section{References}

1. Early J, Hyde R, Baron R. Twenty meter space telescope based on diffractive Fresnel lens. Proceedings of SPIE. The International Society for Optical Engineering, 2004; 5166: 148156.

2. Atcheson P, Stewart C, Domber J, Whiteaker K, Cole J, Spuhler P, Seltzer A, Smith L. MOIRE - Initial demonstration of a transmissive diffractive membrane optic for large lightweight optical telescopes. Proceedings of SPIE - The International Society for Optical Engineering, 2012; 8442: 844221.

3. Atcheson P, Domber J, Whiteaker K, Britten JA, Dixit SN, Farmer B. MOIRE - Ground demonstration of a large aperture diffractive transmissive telescope. Proceedings of SPIE The International Society for Optical Engineering, 2014; 9143: 91431W.

4. Greisukh GI, Efimenko IM, Stepanov SA. Principles of creation of projection and focusing optical systems with diffraction elements. Computer Optics, 1987; 1: 114-116. [In Russian]

5. Greisukh GI, Ezhov EG, Stepanov SA. Aberration properties and performance of a new diffractive-gradient-index high-resolution objective. Applied Optics, 2001; 40(16): 27302735.

6. Zapata-Rodriiguez CJ, Martinez-Corral M, Andres P, Pons A. Axial behavior of diffractive lenses under Gaussian illumination: complex-argument spectral analysis. J. Opt. Soc. Am. A, 1999; 16(10): 2532-2538.

7. Khonina SN, Ustinov AV, Skidanov RV. The binary lens: Study of local foci. Computer Optics, 2011; 35(3): 339-346. [In Russian]

8. Faklis D, Morris GM. Spectral properties of multiorder diffractive lenses. Applied Optics, 1995; 34(14): 2462-2468.

9. Karpeev SV, Alferov SV, Khonina SN, Kudryashov SI. Investigation of the effect of broadband radiation intensity distribution formed by a diffractive optical element. Computer Optics, 2014; 38(4): 689-694. [In Russian]

10. Kotlyar VV, Khonina SN, Soifer VA. Diffraction computation of focusator into longitudinal segment and multifocal lens. Proccedings of SPIE, 1993; 1780: 263-272.

11. Soifer VA, Doskolovich LL, Kazanskiy NL. Multifocal diffractive elements. Optical Engineering, 1994; 33(11): 3610-3615.

12. Dobson SL, Sun P, Fainman Y. Diffractive lenses for chromatic confocal imaging. Applied Optics, 1997; 36(20): 4744-4748.

13. Mait JN, Prather DW, Mirotznik MS. Binary subwavelength diffractive lens design. Optics Letters, 1998; 23(17): 1343-1345.

14. Motogaito A, Hiramatsu K. Fabrication of Binary Diffractive Lenses and the Application to LED Lighting for Controlling Luminosity Distribution. Optics and Photonics Journal, 2013; 3: 67-73. 
15. Greisukh GI, Ezhov EG, Kazin SV, Stepanov SA. Ahromatic diffraction and diffractionrefraction systems of X-Ray range. Computer Optics, 2011; 35(2): 188-195. [In Russian]

16. Farn MW, Goodman JW. Diffractive doublets corrected at two wavelength. J. Opt. Soc. Am. A, 1991; 8(6): 860-867.

17. Andersen G, Tullson D. Broadband antihole photon sieve telescope. Applied Optics, 2007; 46(18): 3706-3708.

18. Zenkevich O, Morgan L. Finite elements and approximations. Moscow: Mir, 1986; 318 p. [In Russian]

19. Zienkiewicz OC, Taylor R. The finite element method. Fifth edition. ButterwothHeinemann, 2000.

20. MSC. Nastran 2004 Reference Manual: Official website of the MSC Corporation. URL: https://simcompanion.mscsoftware.com/resources/sites/MSC/content/meta/DOCUMENT ATION/9000/DOC9188/ secure/refman.pdf

21. Grodzovskij GL, Ivanov VA, Tokarev VV. The mechanics of space flight with the low thrust. Moscow: Nauka, 1966; 704 p. [In Russian]

22. Lebedev VN. Calculation of the low-thrust spacecraft's motion. Moscow: VTS AS USSR, 1968; 108 p. [In Russian]

23. Salmin VV, Chetverikov AS. Management of flat orbital parameters of the geostationary spacecraft with a low-thrust engine. Vestnik SSAU, 2015; 14(4): 92-101. [In Russian]

24. Bellman R. Dynamic programming. Moscow: Inostrannaya literatura, 1960; 400 p. [In Russian] 